\title{
DETERMINATION OF THE ENVIRONMENTAL SENSITIVITY OF SELECTION LINES BY THE SELECTION ENVIRONMENT
}

\author{
J. L. JINKS and V. CONNOLLY* \\ Department of Genetics, University of Birmingham, Birmingham B15 2TT, England
}

Received 21.viii.74

\begin{abstract}
SUMMARY
The parents chosen to continue 10 independent selection lines of Schizophyllum commune over eight successive generations of selection, along with unselected controls, have been retrospectively examined for their response to growth at $15^{\circ}, 20^{\circ}, 25^{\circ}, 30^{\circ}$ and $35^{\circ} \mathrm{C}$. The regression of rate of growth on temperature was essentially linear over the range $15^{\circ}$ to $30^{\circ} \mathrm{C}$ for all lines in all generations as was also the regression of rate of growth on various biological assessments of the environments over the whole temperature range. Either regression, therefore, provided linear regression coefficients which adequately accounted for the relative sensitivities of the lines to temperature in each generation of selection. These measures of environmental sensitivity confirmed our earlier report that selection for high mean performance in a good environment or for low mean performance in a poor environment leads to selections that are more sensitive to environmental variation than selections for high mean performance in a poor environment or for low mean performance in a good environment. These differences in sensitivity emerge as correlated responses during selection and the magnitude of these correlated responses is higher in the good environment than in the poor environment irrespective of the direction of selection.

The environmental sensitivity of selection lines can be modified in either direction as required by either selecting for sensitivity simultaneously with the selection for mean performance or by selecting for mean performance in an above or below average environment. The quality of environments in which artificial selection is usually carried out is likely to have led to high selections with maximum environmental sensitivity and low selections with minimum sensitivity.
\end{abstract}

\section{INTRODUCTION}

IN Schizophyllum commune, selection for an above average phenotype in an above average environment or for a below average phenotype in a below average environment leads to selections that are more sensitive to environmental variation than selection for an above average phenotype in a below average environment or for a below average phenotype in an above average environment (Jinks and Connolly, 1973). This result is predictable from a simple model of gene and environmental action and interaction and is therefore likely to be generally applicable.

Schizophyllum commune can be clonally replicated and stored for long periods at $5^{\circ} \mathrm{C}$. We can, therefore, retrospectively examine any changes in sensitivity to the environment that occurred during the course of selection for the high and low phenotypes in the high and low environments by growing stored cultures of all the parents and controls of each generation of the selections replicated simultaneously over a range of environments.

* Present address: Plant Breeding Department, The Agricultural Institute, Oak Park, Carlow, Eire. 


\section{Materials AND Method}

The material is the 10 selections $2 \mathrm{HP}, 2 \mathrm{LP}, 2 \mathrm{HG}, 2 \mathrm{LG}, 2 \mathrm{H}, 2 \mathrm{~L}, 6 \mathrm{HP}, 6 \mathrm{LP}$, $6 \mathrm{HG}$ and 6LG described by Connolly and Simchen (1973). The numbers (2 and 6) refer to the original wild isolates from which the selections were made, $\mathrm{H}$ and $\mathrm{L}$ are high and low selections for rate of growth and $\mathrm{G}$ and $\mathrm{P}$ are the good or above average $\left(30^{\circ} \mathrm{C}\right)$ and poor or below average $\left(20^{\circ} \mathrm{C}\right.$ ) environments respectively in which the selections were made. Where no environment is specified the selections were made on the basis of performance in both good and poor environments.

The pair of parents chosen to continue each of these 10 selection lines in each of eight successive generations of selection were stored at $5^{\circ} \mathrm{C}$ until the end of the selection programme. Their rates of growth were then determined simultaneously in a single experiment grown in each of the five temperatures, $15^{\circ}, 20^{\circ}, 25^{\circ}, 30^{\circ}$ and $35^{\circ} \mathrm{C}$. Two independent clones of each of the two original isolates, plus 10 unselected progeny of each of these isolates were grown at each temperature as controls. At each temperature two replicate blocks were grown and all cultures were individually randomised within each block. In all 1840 cultures were scored for rate of growth, 368 at each temperature (see Connolly and Simchen, 1973, for further details.)

\section{Results}

In every generation we have four measurements of the rate of growth of each of the selection lines at each of the temperatures. In almost all combinations of lines and generations, rate of growth increases linearly with temperature over the range $15^{\circ}$ to $30^{\circ} \mathrm{C}$ but above $30^{\circ} \mathrm{C}$ it falls off again.

\section{(i) Analysis based on physical measures of the environment}

A linear regression analysis of rate of growth on temperature over the range $15^{\circ}$ to $30^{\circ} \mathrm{C}$ was carried out for each of the 10 selection lines in each of the eight generations of selection. Corresponding analyses were also carried out on the original isolates and the unselected controls. The mean rates of growth ( $\mathrm{mm}$ per 9 days), the linear regression coefficient $\left(\mathrm{mm}\right.$ per ${ }^{\circ} \mathrm{C}$ ) and the percentage of the total sum of squares for rate of growth that is a linear function of temperature are summarised in tables 1,2 and 3. Taking the last first, it is clear that the linear regression coefficient, with few exceptions, accounts for almost all of the effect of temperature on the rate of growth (table 3 ). The exceptions are the advanced generations of selection for low rate of growth in a good environment (2LG and 6LG). The reason is the same for both isolates. The selection for low rate of growth at $30^{\circ} \mathrm{G}$ has been so successful that growth is decreased instead of increased on raising the temperature from $25^{\circ}$ to $30^{\circ} \mathrm{C}$.

The tabulated mean rates of growth (table 1) confirm earlier conclusions (Jinks and Connolly, 1973) that overall there is little difference among the low selections of the same isolate and among the high selections of the same isolate. That is, the environment used for selection does not appear to have had much effect on the performance of the lines when averaged over the four environments although it has a marked effect on the performance in particular environments as has already been described (Jinks and Connolly, 1973). 
TABLE 1

The mean rates of growth ( $\mathrm{mm}$ per 9 days) of the original isolates, the unselected controls and the selection lines in each of eight generations of selection. All are averaged over growth in four environments, $15^{\circ}, 20^{\circ}, 25^{\circ}$, and $30^{\circ} \mathrm{C}$.

Original isolate:

Unselected control:

Selections :

Generation of selection

1
2
3
4
5
6
7
8

\begin{tabular}{|c|c|c|c|c|c|c|c|c|c|}
\hline \multicolumn{6}{|c|}{$\begin{array}{c}\text { Isolate } 2 \\
57.87 \\
53.61\end{array}$} & \multicolumn{4}{|c|}{$\begin{array}{c}\text { Isolate } 6 \\
46 \cdot 94 \\
41 \cdot 50\end{array}$} \\
\hline 2LP & $2 \mathrm{~L}$ & $2 \mathrm{LG}$ & $2 \mathrm{HP}$ & $2 \mathrm{H}$ & $2 \mathrm{HG}$ & $6 \mathrm{LP}$ & $6 \mathrm{LG}$ & $6 \mathrm{HP}$ & $6 \mathrm{LG}$ \\
\hline $50 \cdot 00$ & $47 \cdot 44$ & $47 \cdot 75$ & $58 \cdot 56$ & $58 \cdot 56$ & $59 \cdot 44$ & $31 \cdot 19$ & $35 \cdot 31$ & $50 \cdot 44$ & $50 \cdot 56$ \\
\hline $45 \cdot 06$ & $45 \cdot 44$ & $48 \cdot 06$ & $63 \cdot 38$ & $62 \cdot 25$ & $63 \cdot 25$ & $26 \cdot 69$ & $31 \cdot 88$ & $53 \cdot 75$ & $50 \cdot 00$ \\
\hline $40 \cdot 25$ & $41 \cdot 00$ & $48 \cdot 31$ & $64 \cdot 31$ & $66 \cdot 62$ & 63.94 & $21 \cdot 62$ & $21 \cdot 37$ & $57 \cdot 12$ & $54 \cdot 44$ \\
\hline $38 \cdot 50$ & $35 \cdot 25$ & 43.81 & 64.44 & $65 \cdot 44$ & $66 \cdot 62$ & $22 \cdot 44$ & $22 \cdot 69$ & $54 \cdot 81$ & $56 \cdot 94$ \\
\hline $37 \cdot 69$ & $35 \cdot 50$ & $39 \cdot 06$ & $63 \cdot 56$ & $66 \cdot 81$ & $67 \cdot 19$ & $21 \cdot 56$ & $20 \cdot 06$ & $56 \cdot 44$ & $57 \cdot 19$ \\
\hline $34 \cdot 75$ & 33.88 & 33.06 & $67 \cdot 31$ & $71 \cdot 62$ & $67 \cdot 94$ & $21 \cdot 62$ & $18 \cdot 50$ & $58 \cdot 25$ & $59 \cdot 94$ \\
\hline $31 \cdot 38$ & $31 \cdot 19$ & $34 \cdot 25$ & $68 \cdot 50$ & $72 \cdot 12$ & $68 \cdot 12$ & $22 \cdot 12$ & $17 \cdot 37$ & $62 \cdot 63$ & $60 \cdot 62$ \\
\hline $31 \cdot 00$ & 33.81 & $32 \cdot 37$ & $69 \cdot 31$ & $72 \cdot 75$ & $69 \cdot 00$ & $21 \cdot 25$ & $16 \cdot 63$ & $64 \cdot 25$ & 61.94 \\
\hline
\end{tabular}

TABLE 2

The linear regression coefficients for the regression of rate of growth on temperature $\left(\mathrm{mm}\right.$ per $\left.{ }^{\circ} \mathrm{C}\right)$ of the original isolates, the unselected controls and the selection lines in each of the eight generations of selection

Original isolate:

Unselected control:

Selections :

Generation of selection

1
2
3
4
5
6
7
8

$\begin{array}{lll}3.55 & 2.52 & 2.65 \\ 2.89 & 2.50 & 2.59 \\ 3.19 & 2.00 & 2.47 \\ 2.91 & 1.67 & 1.99 \\ 2.79 & 2.02 & 1.61 \\ 2.83 & 1.57 & 1.21 \\ 2.47 & 1.56 & 0.93 \\ 2.38 & 1.80 & 1.08\end{array}$

$\begin{array}{lll}4 \cdot 31 & 4 \cdot 19 & 4 \cdot 28 \\ 4 \cdot 41 & 4 \cdot 39 & 4 \cdot 60 \\ 4 \cdot 15 & 4 \cdot 80 & 4 \cdot 52 \\ 3 \cdot 83 & 4 \cdot 42 & 4 \cdot 73 \\ 3 \cdot 89 & 4 \cdot 76 & 4 \cdot 64 \\ 4 \cdot 23 & 5 \cdot 01 & 4 \cdot 76 \\ 4 \cdot 19 & 4 \cdot 75 & 4 \cdot 97 \\ 4 \cdot 32 & 4 \cdot 78 & 5 \cdot 19\end{array}$

TABLE 3

$\begin{array}{llll}2.33 & 2.46 & 4.57 & 4.83 \\ 1.53 & 2.02 & 5.14 & 5.00 \\ 1.07 & 1.22 & 5.11 & 5.23 \\ 1.02 & 1.48 & 4.88 & 5.54 \\ 1.28 & 0.96 & 4.76 & 5.27 \\ 1.26 & 0.90 & 4.96 & 5.37 \\ 1.62 & 0.77 & 5.44 & 5.82 \\ 1.96 & 0.79 & 5.53 & 5.67\end{array}$

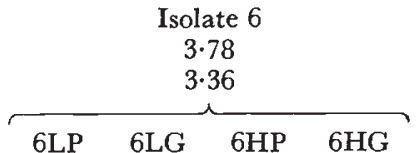

The percentage of the sum of squares for rate of growth accounted for by the linear regression of rate of growth on temperature of the original isolates, the unselected controls and the selection lines in each of the eight generations of selection

Original isolate:

Unselected control:

Selections:

Generation of selection

1
2
3
4
5
6
7
8

\begin{tabular}{|c|c|c|c|c|c|}
\hline $2 \mathrm{LP}$ & $2 \mathrm{~L}$ & $2 \mathrm{LG}$ & $2 \mathrm{HP}$ & $2 \mathrm{H}$ & $2 \mathrm{HG}$ \\
\hline $99 \cdot 8$ & $89 \cdot 2$ & $91 \cdot 3$ & 99.5 & 98.9 & 98.5 \\
\hline $98 \cdot 5$ & $87 \cdot 3$ & $86 \cdot 4$ & $97 \cdot 9$ & $98 \cdot 2$ & $99 \cdot 0$ \\
\hline $99 \cdot 3$ & $81 \cdot 9$ & $95 \cdot 9$ & $96 \cdot 2$ & 99.0 & $98 \cdot 0$ \\
\hline $99 \cdot 2$ & 99.6 & 95.0 & $89 \cdot 1$ & $97 \cdot 9$ & 98.5 \\
\hline $99 \cdot 5$ & 93.5 & $90 \cdot 1$ & 93.0 & $98 \cdot 4$ & $98 \cdot 2$ \\
\hline $99 \cdot 0$ & $87 \cdot 9$ & $80 \cdot 3$ & 95.9 & $98 \cdot 4$ & 96.9 \\
\hline 97.5 & $99 \cdot 2$ & $70 \cdot 1$ & $94 \cdot 5$ & $96 \cdot 2$ & 97.5 \\
\hline 99.9 & 99.0 & $70 \cdot 6$ & 91.6 & $96 \cdot \overline{9}$ & $98 \cdot 4$ \\
\hline
\end{tabular}

$99 \cdot 5$

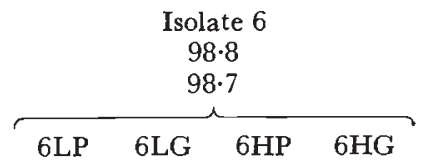

Isolate 2

$\begin{array}{llll}98 \cdot 6 & 97 \cdot 1 & 99 \cdot 1 & 98 \cdot 8 \\ 93.5 & 90 \cdot 2 & 97 \cdot 8 & 98 \cdot 2 \\ 83 \cdot 8 & 96 \cdot 0 & 98 \cdot 1 & 98 \cdot 9 \\ 99 \cdot 4 & 98 \cdot 7 & 98 \cdot 6 & 97 \cdot 2 \\ 98 \cdot 1 & 92 \cdot 3 & 96 \cdot 7 & 96 \cdot 0 \\ 99 \cdot 4 & 91 \cdot 9 & 96 \cdot 1 & 96 \cdot 1 \\ 95 \cdot 8 & 75 \cdot 5 & 94.9 & 99 \cdot 0 \\ 97 \cdot 4 & 61 \cdot 8 & 93.2 & 96 \cdot 9\end{array}$


More important, however, in the present context is the effect on the linear response to temperature.

Relative to the controls all the low selections (2LP, 2L, 2LG, 6LP and $6 \mathrm{LG}$ in table 2) showed a progressive reduction in their linear response to temperature over the eight generations of selection while all the corresponding high selections (2HP, $2 \mathrm{H}, 2 \mathrm{HG}, 6 \mathrm{HP}$ and $6 \mathrm{HG}$ in table 2) showed an increase. These changes in responsiveness between high and low selections will be analysed further in a later paper. It is more relevant to the present discussion that the changes while consistent in direction, are not uniform in magnitude over all the low or all the high selections from the same isolate. They conform to the pattern previously reported (Jinks and Connolly, 1973). As selection proceeds the low selections in the good environment (LG) and the high selections in the poor environment (HP) become relatively less sensitive to temperature than the corresponding low selections in a poor environment (LP) and the high selections in the good environment (HG). At the same time the selections based on performance in both environments ( $\mathrm{L}$ and $\mathrm{H}$ ) become intermediate in their sensitivities.

Elsewhere (Jinks and Connolly, 1973) we have argued that these changes in the sensitivity of rate of growth to temperature are not random but correlated responses to the selections for high and low rates of growth and that the magnitude of the correlation is determined by the temperature upon which the selections were based. The correctness of this interpretation can now be demonstrated directly because we can calculate the magnitude of the correlated response for each selection in each environment over the eight generations of selection. The response of rate of growth to selection over the eight generations can be assessed from the means in table 1 and the correlated response in sensitivity of rate of growth to temperature from the regression coefficients in table 2. The magnitude of the correlated response for each selection line can be expressed, therefore, as the regression over the eight generations of selection of these regression coefficients on to the corresponding means. If this regression is essentially linear it will measure the change in sensitivity to temperature per unit response of rate of growth to selection.

For the four selections 2LP, 2LG, 2HP and 2HG over 90 per cent of the variation in sensitivity can be accounted for by a linear regression on the response of the mean rate of growth to selection. Furthermore, there is no significant difference between the high and low selections in the same environment for this linear regression (the probability equals $0.8-0.9$ and $0.3-0.2$ for $\mathrm{P}$ and $\mathrm{G}$ environments, respectively), but there is a highly significant difference between environments $(P<0.001)$. The best fitting regression in the poor environment (2LP and $2 \mathrm{HP}$ ) has a linear coefficient of $0.0479 \pm 0.0039$ while in the good environment (2LG and $2 \mathrm{HG}$ ) it has a coefficient of $0 \cdot 1133 \pm 0 \cdot 0035$. Selection whether for high or low rate of growth, therefore, leads to more than twice the correlated change in the sensitivity of the selection lines to temperature when the selection is based on performance in the good environment as when based on performance in the poor environment. The selections of isolate 2 based on performance in both environments ( $2 \mathrm{~L}$ and $2 \mathrm{H}$ ) give an intermediate value for the regression of $0.0884 \pm 0.0038$. Selection for high rate of growth in a good environment therefore gives the most sensitive and selection for low rate of growth in a good environment the least sensitive of all the selection lines. Analysis of the corresponding four selections of isolate 6 gives essentially the same result. 
The difference between the poor and good environments is small but nonetheless significant $(P=0.02-0.03)$ the linear coefficients being $0 \cdot 1035 \pm 0.0043$ for the poor environment $(6 \mathrm{LP}$ and $6 \mathrm{HP})$ and $0 \cdot 1166 \pm 0.0035$ for the good environment (6LG and $6 \mathrm{HG})$.

\section{(ii) Analyses based on biological measures of the environment}

In the analyses described so far the $35^{\circ} \mathrm{C}$ environment has been excluded so that a linear regression of rate of growth on temperature would be an adequate summary of the response of rate of growth to temperature. This simplification is not a prerequisite for analysing these data and it does not influence the conclusions. The non-linearity at higher temperatures can be accommodated as in previous papers by using a biological measure of the environmental differences and in these data we have a choice of such measures. All give essentially the same result and none differs from the result obtained in the earlier analyses in which temperature was the measure of the environment.

\section{TABLE 4}

The results of regressing rate of growth on to temperature over the range $15^{\circ}$ to $30^{\circ} \mathrm{C}$ and on to a biological measure of the environment (the rate of growth of the unselected controls over the temperature range $15^{\circ}$ to $35^{\circ} \mathrm{C}$ ) for the last generation of the selections of isolate 2 . The linear regression coefficient (b) the percentage of the sum of squares accounted for by the linear regression and rescaled values for $\mathrm{b}$ based on a unit value for the original isolate are listed:

\begin{tabular}{|c|c|c|c|c|c|c|}
\hline \multirow[b]{2}{*}{ Lines } & \multicolumn{3}{|c|}{ Regression on to temperature } & \multicolumn{3}{|c|}{ Regression on to control } \\
\hline & $b$ & Linear S.S. & Scaled $b$ & $b$ & Linear S.S. & Scaled $b$ \\
\hline Original & & & & & & \\
\hline isolate & $3 \cdot 94$ & $99 \cdot 5$ & $1 \cdot 00$ & $0 \cdot 98$ & $96 \cdot 8$ & $1 \cdot 00$ \\
\hline 2LP & $2 \cdot 38$ & $99 \cdot 9$ & $0 \cdot 60$ & $0 \cdot 58$ & $98 \cdot 8$ & $0 \cdot 59$ \\
\hline 2L & $1 \cdot 80$ & $99 \cdot 0$ & 0.46 & $0 \cdot 43$ & $88 \cdot 7$ & $0 \cdot 44$ \\
\hline 2LG & $1 \cdot 08$ & $70 \cdot 6$ & 0.27 & $0 \cdot 28$ & $76 \cdot 3$ & $0 \cdot 29$ \\
\hline $2 \mathrm{HP}$ & $4 \cdot 32$ & $91 \cdot 6$ & $1 \cdot 09$ & $1 \cdot 07$ & $93 \cdot 5$ & $1 \cdot 09$ \\
\hline $2 \mathrm{H}$ & $4 \cdot 78$ & $96 \cdot 9$ & $1 \cdot 21$ & $1 \cdot 18$ & $98 \cdot 7$ & $1 \cdot 23$ \\
\hline $2 \mathrm{HG}$ & $5 \cdot 19$ & $98 \cdot 4$ & $1 \cdot 32$ & $1 \cdot 25$ & $94 \cdot 9$ & $1 \cdot 28$ \\
\hline
\end{tabular}

There is no point, therefore, in repeating all the analyses using a biological measure; however, to illustrate the closeness of the agreement, the results of these alternative analyses, when applied to the last generation of the selections of isolate 2, have been summarised in table 4 . The regression coefficients and the percentages of the total sum of squares accounted for by the linear regression are tabulated for the regression on temperature over the range $15^{\circ}$ to $30^{\circ} \mathrm{C}$ and for the regression on one of the biological measures of the environmental values, namely, the mean rates of growth of the 10 unselected lines of isolate 2 when grown at $15^{\circ}, 20^{\circ}, 25^{\circ}, 30^{\circ}$ and $35^{\circ} \mathrm{C}$. Although the latter analysis includes the data for $35^{\circ} \mathrm{C}$ the two analyses show little difference in either the overall percentage of the total sum of squares accounted for by the linear regressions or in the variation in the percentages among the selection lines. The regression coefficients themselves are, of course, on different scales, and they are not, therefore, directly comparable. But it is immediately obvious that the linear responses of the six selections and the original isolate to environmental variation are identically ranked by the two analyses. The two sets of regression coefficients can be converted to the 
same scale by making the regression coefficient of one of the lines unity in both analyses by dividing all the regression coefficients by the observed coefficient for that line. The results of such a re-scaling based on taking the regression coefficient of the original isolate 2 as unity are shown in table 4 . The close agreement of the two methods of measuring sensitivity to environmental variation in these data is now quite clear.

\section{Discussion}

In this paper we have confirmed that both high and low selections differ in their sensitivity to environmental differences according to whether the selections were based on assessments of their performance in a good or in a poor environment. We have also shown how these differences in sensitivity emerge during selection as a correlated response that is greater in magnitude where a good environment has been used for selection than when a poor environment has been used.

During a selection programme designed to change mean performance one can, therefore, influence the environmental sensitivity of the resulting selection lines in one of two ways. One can monitor the sensitivity of the selection lines as well as their mean performance and incorporate into the selection programme simultaneous selection for the desired levels of sensitivity and mean performance. This approach has been discussed in detail by Perkins and Jinks (1971) and a selection programme based on it has been carried out by our colleague, Dr R. Brumpton (unpublished Ph.D. thesis, 1973). Alternatively, one can select for mean performance in an environment which has been chosen to produce the desired level of correlated responses in environmental sensitivity. An environment may be chosen which will lead to greater than or smaller than average environmental sensitivities in the selection lines, as required. This alternative approach can be less demanding and in our experience it can be equally effective.

Artificial selection has in general been based on assessments of performance made in environments which are good relative to those in which the material is usually raised. Selection in such environments will maximise the environmental sensitivity of high selections and minimise that of low selections. It is, therefore, more likely to lead to the high positive correlations between mean performance and sensitivity that have been so widely reported for crop plants. If these are not the optimal combinations of mean performance and environmental sensitivity one obvious step that might be taken is to select on the basis of performance in less good environments.

Acknowledgments.-We are indebted to $\mathrm{Mr}$ P. J. Jinks for assisting with the statistical analysis and to Professor K. Mather for his helpful comments during the preparation of this paper.

\section{REFERENCES}

CONNOLly, V., AND simchen, G. 1973. Two-environment selection with inbreeding in Schizophyllum commune. Genet. Res. Camb., 22, 1-12.

JINKS, J. L., AND CONNOLLY, v. 1973. Selection for specific and general response to environmental differences. Heredity, 30, 33-40.

PERKINS, JEAN M., AND JINKS, J. L. 1971. Specificity of the interaction of genotypes with contrasting environments. Heredity 26, 463-474. 